\title{
Existence of positive solutions to periodic boundary value problems with sign-changing Green's function
}

\author{
Shengren Zhong ${ }^{1}$ and Yukun $\mathrm{An}^{2^{*}}$
}

\footnotetext{
* Correspondence: anykna@nuaa. edu.cn

${ }^{2}$ Department of Mathematics, Nanjing University of Aeronautics and Astronautics Nanjing, 210016, PR China

Full list of author information is available at the end of the article
}

\section{Abstract}

This paper deals with the periodic boundary value problems

$$
\left\{\begin{array}{l}
u^{\prime \prime}+\rho^{2} u=f(u), \quad 0<t<T, \\
u(0)=u(T), u^{\prime}(0)=u^{\prime}(T)
\end{array}\right.
$$

where $0<\rho \leq \frac{3 \pi}{2 T}$ is a constant and in which case the associated Green's function may changes sign. The existence result of positive solutions is established by using the fixed point index theory of cone mapping.

Keywords: periodic boundary value problem, positive solution, sign-changing Green's function, cone, fixed point theorem

\section{Introduction}

The periodic boundary value problems

$$
\left\{\begin{array}{l}
u^{\prime \prime}+a(t) u=f(t, u), \quad 0<t<T \\
u(0)=u(T), u^{\prime}(0)=u^{\prime}(T)
\end{array}\right.
$$

where $f$ is a continuous or $L^{1}$-Caratheodory type function have been extensively studied. A very popular technique to obtain the existence and multiplicity of positive solutions to the problem is Krasnosel'skii's fixed point theorem of cone expansion/ compression type, see for example [1-4], and the references contained therein. In those papers, the following condition is an essential assumptions:

$(A)$ The Green function $G(t, s)$ associated with problem $(1)$ is positive for all $(t, s) \in$ $[0, T] \times[0, T]$.

Under condition $(A)$, Torres get in [4] some existence results for (1) with jumping nonlinearities as well as (1) with a repulsive or attractive singularity, and the authors in [3] obtained the multiplicity results to (1) when $f(t, u)$ has a repulsive singularity near $x$ $=0$ and $f(t, u)$ is super-linear near $x=+\infty$. In [2], a special case, $a(t) \equiv m^{2}$ and $m \in\left(0, \frac{\pi}{T}\right)$, was considered, the multiplicity results to (1) are obtained when the nonlinear term $f(t, u)$ is singular at $u=0$ and is super-linear at $u=\infty$.

Recently, in [5], the hypothesis $(A)$ is weakened as

$(B)$ The Green function $G(t, s)$ associated with problem (1) is nonnegative for all $(t, s) \in[0, T] \times[0, T]$ but vanish at some interior points.

(c) 2011 Zhong and An; licensee Springer. This is an Open Access article distributed under the terms of the Creative Commons Attribution License (http://creativecommons.org/licenses/by/2.0), which permits unrestricted use, distribution, and reproduction in any medium, provided the original work is properly cited. 
By defining a new cone, in order to apply Krasnosel'skii's fixed point theorem, the authors get an existence result when $f(t, u)=g(t) \bar{f}(u)$ and $\bar{f}(u)$ is sub-linear at $u=0$ and $u=\infty$ or $\bar{f}(u)$ is super-linear at $u=0$ and $u=\infty$ with $\bar{f}(u)$ is convex and nondecreasing.

In [6], the author improve the result of [5] and prove the existence results of at least two positive solutions under conditions weaker than sub- and super-linearity.

In [7], the author study (1) with $f(t, u)=\lambda b(t) f(u)$ under the following condition:

(C) The Green function $G(t, s)$ associated with problem (1) changes sign andmin $\operatorname{mi}_{t \in[0, T]} \int_{0}^{T} G^{-}(t, s) d s=m^{*}>0$ where $G^{-}$is the negative part of $G$.

Inspired by those papers, here we study the problem:

$$
\left\{\begin{array}{l}
u^{\prime \prime}+\rho^{2} u=f(u), \quad 0<t<T, \\
u(0)=u(T), u^{\prime}(0)=u^{\prime}(T),
\end{array}\right.
$$

where $0<\rho \leq \frac{3 \pi}{2 T}$ is a constant and the associated Green's function may changes sign. The aim is to prove the existence of positive solutions to the problem.

\section{Preliminaries}

Consider the periodic boundary value problem

$$
\left\{\begin{array}{l}
u^{\prime \prime}+\rho^{2} u=e(t), \quad 0<t<T, \\
u(0)=u(T), u^{\prime}(0)=u^{\prime}(T),
\end{array}\right.
$$

where $0<\rho \leq \frac{3 \pi}{2 T}$ and $e(t)$ is a continuous function on [0,T]. It is well known that the solutions of (3) can be expressed in the following forms

$$
u(t)=\int_{0}^{T} G(t, s) e(s) d s,
$$

where $G(t, s)$ is Green's function associated to (3) and it can be explicitly expressed

$$
G(t, s)=\left\{\begin{array}{l}
\frac{\sin \rho(t-s)+\sin \rho(T-t+s)}{2 \rho(1-\cos \rho T)}, 0 \leq s \leq t \leq T, \\
\frac{\sin \rho(s-t)+\sin \rho(T-s+t)}{2 \rho(1-\cos \rho T)}, 0 \leq t \leq s \leq T .
\end{array}\right.
$$

By direct computation, we get

$$
\frac{\sin \rho T}{2 \rho(1-\cos \rho T)} \leq G(t, s) \leq \frac{\sin \frac{\rho T}{2}}{\rho(1-\cos \rho T)}=\max _{t, s \in[0, T]} G(t, s),
$$

and

$$
G(t, s)<0
$$

for $|t-s|<\frac{T}{2}-\frac{\pi}{2 \rho}$ when $\frac{\pi}{T} \leq \rho \leq \frac{3 \pi}{2 T}$, and

$$
\begin{aligned}
& g(t)=\int_{0}^{T} G(t, s) d s=\frac{1}{\rho^{2}}, t \in[0, T], \\
& \min _{t \in[0, T]} \frac{\int_{0}^{T} G^{+}(t, s) d s}{\int_{0}^{T} G^{-}(t, s) d s}=\frac{1}{1-\sin \frac{\rho T}{2}},
\end{aligned}
$$

where $G^{+}$and $G^{-}$are the positive and negative parts of $G$. 
We denote

$$
\sigma=\frac{1}{\rho^{2} \max _{t, s \in[0, T]} G(t, s)}=\frac{2 \sin \frac{\rho T}{2}}{\rho},
$$

and

$$
\gamma= \begin{cases}+\infty, & 0 \leq \rho \leq \frac{\pi}{T}, \\ \frac{1}{1-\sin \frac{\rho T}{2}}, & \frac{\pi}{T}<\rho \leq \frac{3 \pi}{2 T} .\end{cases}
$$

Let $E$ denote the Banach space $C[0, T]$ with the norm $\|u\|=\max _{t \in[0, T]}|u(t)|$.

Define the cone $K$ in $E$ by

$$
K=\left\{u \in E: u \geq 0, \int_{0}^{T} u(s) d s \geq \sigma\|u\|\right\} .
$$

We know that $\sigma=\frac{\sin \frac{\rho T}{2}}{\frac{\rho}{2}}<T$ and therefore $K \neq \varnothing$. For $r>0$, let $K_{r}=\{u \in K:\|u\|<$ $r\}$, and $\partial K_{r}=\{u \in K:\|u\|=r\}$, which is the relative boundary of $K_{r}$ in $K$.

To prove our result, we need the following fixed point index theorem of cone mapping.

Lemma 1 (Guo and Lakshmikantham [8]). Let $E$ be a Banach space and let $K \subset E$ be a closed convex cone in $E$. Let $L: K \rightarrow K$ be a completely continuous operator and let $i\left(L, K_{r}, K\right)$ denote the fixed point index of operator $L$.

(i) If $\mu L u \neq u$ for any $u \in \partial K_{r}$ and $0<\mu \leq 1$, then

$$
i\left(L, K_{r}, K\right)=1 .
$$

(ii) If $\inf _{u \in \partial K_{r}}|| L u \|>0$ and $\mu L u \neq u$ for any $u \in \partial K_{r}$ and $\mu \geq 1$, then

$$
i\left(L, K_{r}, K\right)=0 .
$$

\section{Existence result}

We make the following assumptions: $(H 1) f:[0,+\infty) \rightarrow[0,+\infty)$ is continuous;

$(H 2) 0 \leq m=\inf _{u \in[0,+\infty]} f(u)$ and $M=\sup _{u \in[0,+\infty)} f(u) \leq+\infty$;

(H3) $\frac{M}{m} \leq \gamma$, when $m=0$ we define $\frac{M}{m}=+\infty$.

To be convenience, we introduce the notations:

$$
f_{0}=\lim _{u \rightarrow 0} \frac{f(u)}{u} \text { and } f_{\infty}=\lim _{u \rightarrow \infty} \frac{f(u)}{u},
$$

and suppose that $f_{0}, f_{\infty} \in[0, \infty]$.

Define a mapping $L: K \rightarrow E$ by

$$
L u(t)=\int_{0}^{T} G(t, s) f(u(s)) d s, t \in[0, T] .
$$

It can be easily verified that $u \in K$ is a fixed point of $L$ if and only if $u$ is a positive solution of (2).

Lemma 2. Suppose that $\left(H_{1}\right),\left(H_{2}\right)$ and $\left(H_{3}\right)$ hold, then $L: E \rightarrow E$ is completely continuous and $L(K) \subseteq K$. 
Proof Let $u \in K$, then in case of $\gamma=+\infty$, since $G(t, s) \geq 0$, we have $L u(t) \geq 0$ on [0, $T]$; in case of $\gamma<+\infty$, we have

$$
\begin{aligned}
L u(t) & =\int_{0}^{T} G(t, s) f(u(s)) d s \\
& =\int_{0}^{T}\left(G^{+}(t, s)-G^{-}(t, s)\right) f(u(s)) d s \\
& \geq \int_{0}^{T}\left(G^{+}(t, s) m-G^{-}(t, s) M\right) d s \\
& =m \int_{0}^{T}\left(G^{+}(t, s)-\frac{M}{m} G^{-}(t, s)\right) d s \\
& \geq m \int_{0}^{T}\left(G^{+}(t, s)-\gamma G^{-}(t, s)\right) d s \\
& \geq 0 .
\end{aligned}
$$

On the other hand,

$$
\begin{aligned}
\int_{0}^{T} L u(t) d t & =\int_{0}^{T} \int_{0}^{T} G(t, s) f(u(s)) d s d t \\
& =\int_{0}^{T} f(u(s)) \int_{0}^{T} G(t, s) d t d s \\
& \geq \frac{1}{\rho^{2}} \int_{0}^{T} f(u(s)) d s .
\end{aligned}
$$

and

$$
L u(t)=\int_{0}^{T} G(t, s) f(u(s)) d s \leq \max _{t, s \in[0, T]} G(t, s) \int_{0}^{T} f(u(s)) d s
$$

for $t \in[0, T]$. Thus,

$$
\int_{0}^{T} L u(t) d t \geq \sigma \max _{t \in[0, T]}|L u(t)|
$$

i.e., $L(K) \subseteq K$. A standard argument can be used to show that $L: E \rightarrow E$ is completely continuous.

Now we give and prove our existence theorem:

Theorem 3. Assume that $\left(H_{1}\right),\left(H_{2}\right)$ and $\left(H_{3}\right)$ hold. Furthermore, suppose that $f_{0}>\rho^{2}$ and $f_{\infty}<\rho^{2}$ in case of $\gamma=+\infty$. Then problem (2) has at least one positive solution.

Proof Since $f_{0}>\rho^{2}$, there exist $\varepsilon>0$ and $\xi>0$ such that

$$
f(u) \geq\left(\rho^{2}+\varepsilon\right) u, \quad \text { forall } u \in[0, \xi] .
$$

Let $r \in(0, \xi)$, then for every $u \in \partial K_{r}$, we have

$$
\begin{aligned}
T\|L u\| & \geq \int_{0}^{T} L u(t) d t \\
& =\int_{0}^{T} f(u(s)) \int_{0}^{T} G(t, s) d t d s \\
& \geq \frac{1}{\rho^{2}} \int_{0}^{T} f(u(s)) d s \\
& \geq \frac{\rho^{2}+\varepsilon}{\rho^{2}} \int_{0}^{T} u(s) d s \\
& \geq \frac{\left(\rho^{2}+\varepsilon\right) \sigma r}{\rho^{2}}>0 .
\end{aligned}
$$


Hence, $\inf _{u \in \partial K_{r}}|| L u \|>0$. Next, we show that $\mu L u \neq u$ for any $u \in \partial K_{r}$ and $\mu \geq 1$. In fact, if there exist $u_{0} \in \partial K_{r}$ and $\mu_{0} \geq 1$ such that $\mu_{0} L u_{0}=u_{0}$, then $u_{0}(t)$ satisfies

$$
\left\{\begin{array}{l}
u_{0}^{\prime \prime}(t)+\rho^{2} u_{0}(t)=\mu_{0} f\left(u_{0}(t)\right), 0<t<T \\
u_{0}(0)=u_{0}(T), u_{0}^{\prime}(0)=u_{0}^{\prime}(T)
\end{array}\right.
$$

Integrating the first equation in (5) from 0 to $T$ and using the periodicity of $u_{0}(t)$ and (4), we have

$$
\begin{aligned}
\left.\rho^{2} \int_{0}^{T} u_{0}(t)\right) d t & =\mu_{0} \int_{0}^{T} f\left(u_{0}(t)\right) d s \\
& \geq\left(\rho^{2}+\varepsilon\right) \int_{0}^{T} u_{0}(t) d t
\end{aligned}
$$

Since $\left.\int_{0}^{T} u_{0}(t)\right) d t \geq \sigma\left\|u_{0}\right\|>0$, we see that $\rho^{2} \geq\left(\rho^{2}+\varepsilon\right)$, which is a contradiction. Hence, by Lemma 1 , we have

$$
i\left(L, K_{r}, K\right)=0 \text {. }
$$

On the other hand, since $f_{\infty}<\rho^{2}$, there exist $\varepsilon \in\left(0, \rho^{2}\right)$ and $\zeta>0$ such that

$$
f(u) \leq\left(\rho^{2}-\varepsilon\right) u, \quad \text { forall } u \geq \zeta .
$$

Set $C=\max _{0 \leq u \leq \zeta}\left|f(u)-\left(\rho^{2}-\varepsilon\right) u\right|+1$, it is clear that

$$
f(u) \leq\left(\rho^{2}-\varepsilon\right) u+C, \quad \text { forall } u \geq 0 .
$$

If there exist $u_{0} \in K$ and $0<\mu_{0} \leq 1$ such that $\mu_{0} L u_{0}=u_{0}$, then (5) is valid. Integrating again the first equation in (5) from 0 to $T$, and from (7), we have

$$
\begin{aligned}
\left.\rho^{2} \int_{0}^{T} u_{0}(t)\right) d t & =\mu_{0} \int_{0}^{T} f(u(t)) d t \\
& \leq\left(\rho^{2}-\varepsilon\right) \int_{0}^{T} u_{0}(t) d t+C .
\end{aligned}
$$

Therefore, we obtain that

$$
\frac{C}{\varepsilon} \geq \int_{0}^{T} u_{0}(t) d t \geq \sigma\left\|u_{0}\right\|
$$

i.e.,

$$
\left\|u_{0}\right\| \leq \frac{C}{\sigma \varepsilon} .
$$

Let $R>\max \left\{\frac{C}{\sigma \varepsilon}, \xi\right\}$, then $\mu L u \neq u$ for any $u \in \partial K_{R}$ and $0<\mu \leq 1$. Therefore, by Lemma 1 , we get

$$
i\left(L, K_{R}, K\right)=1 \text {. }
$$

From (6) and (9) it follows that

$$
i\left(L, K_{R} \backslash \bar{K}_{r}, K\right)=i\left(L, K_{R}, K\right)-i\left(L, K_{r}, K\right)=1 .
$$

Hence, $L$ has a fixed point in $K_{R} \backslash \bar{K}_{r}$, which is the positive solution of (2). 
Remark 4. Theorem 3 contains the partial results of [4-7] obtained in case of positive Green's function, vanishing Green's function and sign-changing Green's function, respectively.

\section{An example}

Let $0 \neq q<1$ be a constant, $h$ be the function:

$$
h(x)= \begin{cases}1, & x \geq 0 \\ 0, & x<0\end{cases}
$$

and let

$$
f(u)=1+h\left(\frac{\pi}{T}-\rho\right) u^{q}+\left(1-h\left(\frac{\pi}{T}-\rho\right)\right) \frac{2 \sin \frac{\rho T}{2}}{\pi\left(1-\sin \frac{\rho T}{2}\right)} \arctan u .
$$

By the direct calculation, we get $m=1$ and $M=\gamma$, and $f_{0}=\infty$ and $f_{\infty}=0$ in case of $\gamma$ $=+\infty$. Consider the following problem

$$
\left\{\begin{array}{l}
u^{\prime \prime}+\rho^{2} u=f(u), \quad 0<t<T, \\
u(0)=u(T), u^{\prime}(0)=u^{\prime}(T),
\end{array}\right.
$$

where $0<\rho \leq \frac{3 \pi}{2 T}$ is a constant. We know that the conditions of Theorem 3 hold for the problem (10) and therefore, (10) have at least one positive solution from Theorem 3.

\section{Acknowledgements}

The authors are very grateful to the anonymous referee whose careful reading of the manuscript and valuable comments enhanced presentation of the manuscript.

\section{Author details}

'Department of Engineering Technology, Wuwei Occupational College Wuwei, 733000, Gansu, PR China ²Department of Mathematics, Nanjing University of Aeronautics and Astronautics Nanjing, 210016, PR China

\section{Authors' contributions}

YA conceived of the study, and participated in its coordination. SZ drafted the manuscript. All authors read and approved the final manuscript.

\section{Competing interests}

The authors declare that they have no competing interests.

Received: 27 January 2011 Accepted: 27 July 2011 Published: 27 July 2011

\section{References}

1. Graef, JR, Kong, L, Wang, H: Existence, multiplicity and dependence on a parameter for a periodic boundary value problem. J Differ Equ. 245, 1185-1197 (2008). doi:10.1016/j.jde.2008.06.012

2. Jiang, D, Chu, J, O'Regan, D, Agarwal, RP: Multiple positive solutions to supperlinear periodic boundary value problem with repulsive singular equations. J Math Anal Appl. 286, 563-576 (2003). doi:10.1016/S0022-247X(03)00493-1

3. Jiang, D, Chu, J, Zhang, M: Multiplicity of positive periodic solutions to supperlinear repulsive singular equations. J Differ Equ. 210, 282-320 (2005)

4. Torres, PJ: Existence of one-signed periodic solutions of some second-order differential equations via a Krasnoselskii fixed point theorem. J Differ Equ. 190, 643-662 (2003). doi:10.1016/S0022-0396(02)00152-3

5. Graef, JR, Kong, L, Wang, H: A periodic boundary value problem with vanishing Green's function. Appl Math Lett. 21, 176-180 (2008). doi:10.1016/j.aml.2007.02.019

6. Webb, TRL: Boundary value problems with vanishing Green's function. Commun Appl Anal. 13(4), $587-596$ (2009)

7. Ma, R: Nonlinear periodic boundary value problems with sign-changing Green's function. Nonlinear Anal. 74, 1714-1720 (2011). doi:10.1016/j.na.2010.10.043

8. Guo, D, Lakshmikantham, V: Nonlinear Problems in Abstract Cones. Academic Press, New York (1988)

doi:10.1186/1687-2770-2011-8

Cite this article as: Zhong and An: Existence of positive solutions to periodic boundary value problems with sign-changing Green's function. Boundary Value Problems 2011 2011:8. 